\title{
Bioanalysis
}

\section{7th Annual Symposium on Clinical and Pharmaceutical Solutions through Analysis}

\section{7th Annual Symposium on Clinical \& Pharmaceutical Solutions through Analysis, Renaissance Shanghai Pudong Hotel, Shanghai, China, 20-23 April 2016}

The 7th Annual Shanghai Symposium on Innovative Approaches to Reduce Attrition and Predict Clinical Outcomes (CPSA Shanghai 2016) was held on 20-23 April 2016 in Renaissance Shanghai Pudong Hotel, Shanghai, China. The meeting was featured with highly interactive events including diversified symposia, round table discussions, workshops, poster sessions and conference awards. There were over 220 participants from more than ten countries, with 61 oral presentations and 29 posters presented. In addition, the meeting included one preconference workshop and three joint sessions held with bioanalytical experts from local communities.

First draft submitted: 3 August 2016; Accepted for publication: 8 August 2016; Published online: 9 September 2016

Keywords: bioanalysis $\bullet$ biomarker $\bullet$ biomolecule $\bullet$ clinical outcome $\bullet$ CPSA, drug development

Kelly Dong of GlaxoSmithKine (Shanghai, China) served as the 7th Annual Shanghai Symposium on Innovative Approaches to Reduce Attrition and Predict Clinical Outcomes (CPSA Shanghai 2016) Program Chair. The CPSA founder, Mike Lee of Milestone Development Services (Newtown, PA, USA), opened the conference by welcoming the audience and giving thanks to the organizing committee and the volunteers. The theme of the CPSA Shanghai 2016 was 'Innovative approaches to reduce attrition and predict clinical outcomes' [1]. The program features updated perspectives and experiences on clinical and pharmaceutical analyses that meet specific regulations from the US FDA or Clinical Laboratories Improvement Amendments (CLIA) with highest level of performance (e.g., accuracy, sensitivity, throughput and cost).

Valleriu Damian of GlaxoSmithKline (Hertfordshire, UK) gave a plenary lecture on 'Quantitative system pharmacology - a key approach to reduce attrition in drug discovery and development.'

Quantitative system pharmacology, through revelation of quantitative results and correlations, is shown to provide an effective approach to reduce attrition in drug discovery and accelerate translational research.

Nathan Yates of University of Pittsburgh (La Jolla, CA, USA) did another plenary lecture on 'Differential mass spectroscopy: biomarker discovery form basic research to the clinic.' The differential MS has been applied to the drug responsive markers cerebrospinal fluid proteomics and measuring protein-protein interactions. It is a new paradigm for PD markers discovery and can reveal aging similarities and differences between strains of mice, and mice housed under different conditions.

The symposium included over 15 sessions and covered a broad range of topics. This
Tianyi (Tee) Zhang ${ }^{*, 1}$, Li Wang' ${ }^{2}$ Naidong Weng ${ }^{3}$, Kelly Dong ${ }^{4}$, Gary Valaskovic ${ }^{5}$ \& Mike Lee ${ }^{6}$

'Frontage Laboratories, No.13 Building, 67 Libing Road, Zhangjiang Hi-Tech Park, Shanghai 201203, China

${ }^{2}$ Frontage Laboratories, Shanghai, China 3Janssen, Pharmaceutical companies of Johnson \& Johnson, USA

${ }^{4}$ GlaxoSmithKine, Shanghai, China ${ }^{5}$ New Objective, Woburn, MA, USA ${ }^{6}$ Milestone Development Services, Newtown, PA, USA

*Author for correspondence:

tianyizhang@frontagelab.com.cn 
conference report summarizes some of the highlights from the contributed sessions.

\section{Preconference workshop sponsored by SCIEX}

Kerong Zhang of SCIEX (Shanghai, China) presented 'Advantages of novel data acquisition strategies for accurate metabolite identification.' The report noted the advantages of the triple TOF 6600 platform, the sequential window acquisition of all theoretical fragmentions (SWATH) data collection strategies and research version of Metabolite Pilot in supporting the drug metabolism investigation. These techniques could enable streamlining of quantitative and qualitative drug metabolism and PK (DMPK) studies.

Shaw Xia of SCIEX (Shanghai, China) lectured on 'Practical considerations of large molecule bioanalysis in complex matrices.' In comparison with ELISA, the LC-MS/MS could be used to analyze the biologics/biosimilar drug with simplified sample preparation, higher sensitivity and selectivity, and save time and money. Several case studies using LC-MS/MS for quantitative determination of biologics/biosimilar drug were presented.

\section{Innovative approaches to reduce attrition}

Naidong Weng of Janssen Research \& Development (Spring House, PA, USA) presented 'Endogenous biomarkers for drug-drug interaction and recommendation of bioanalysis of $4 \beta$-hydroxycholesterol for P450 $3 \mathrm{~A} 4 / 5$ DDI.' The report showed the advantage of using endogenous biomarkers for drug-drug interaction studies, including advantages in sample handling, quantification, specificity, stability and sensitivity. Recommendation for bioanalysis of $4 \beta$-hydroxycholesterol for P450 3A4/5 drug-drug interaction was presented. However, there were still challenges; for example, only very few endogenous biomarkers are available for $3 \mathrm{~A} 4 / 5$. Broader collaborations and industrial consortium on development of robust and specific bioanalytical assays for biomarkers are needed.

Joseph Bower of Covance (Chantilly, VA, USA) gave a presentation on 'Bioanalytical strategies to help drive drug development.' Bioanalytical data do not directly impact rates of attrition for clinical studies. However, it is a powerful tool that can be used throughout drug development process. Bioanalytical data can help improve selection of candidate drug molecules to progress in nonclinical evaluation of safety/toxicity in relevant nonclinical species. Bioanalytical data can also help determine the optimal therapeutic dose and assess safety and efficacy of new therapies in clinical trials.

Mary Hu of Miracoge (Shanghai, China) lectured on 'Bioanalytical strategy for antibody drug conjugates
(ADCs).' The report noted that antibody drug conjugates (ADCs) are a class of complex cancer therapeutics showing great potentials during in vitro and in vivo studies. In ADC development, appropriately strategizing ADC bioanalysis is essential, like choosing analytes relevant to safety and efficacy, starting as early as possible, generating and maintaining critical reagents, ensuring reliability of chosen assays and saving time and cost.

\section{Small-molecule bioanalysis}

Xiao Ding of Genentech (Shanghai, China) presented 'Application of LC-AMS for the determination of absolute bioavailability of taselisib (GDC-0032) in human.' GDC-0032 is a potent, novel, oral and selective inhibitor of PI3K $\alpha, \gamma$ and $\delta$ isoforms (MW: 460.5). It was determined using a qualified UPLC-AMS method with the standard curve and recovery factor models. The latter was used for sample analysis. The measured absolute bioavailability of GDC-0032 was $57.4 \%$ (standard deviation: 0.128 ).

Hongliang Jiang of HuaZhong University of Science and Technology (Hubei Province, China) lectured on 'Bioanalytical challenges and strategies for analysis of unstable small molecules.' The report noted the factors affecting stability of small molecules in biological matrix and shared several strategies, including general strategies for matrix and anticoagulant selection, control (temperature and light) and prevention of intermolecular conversion, and specific approaches like $\mathrm{pH}$ control, addition of enzyme inhibitors, addition of antioxidants and derivatization.

Luke Bi of Covance (Shanghai, China) presented 'Addressing matrix effect issue in clinical bioanalysis.' Matrix effect could contribute to assay variability and could come from disease population, comedication and matrix in difference races. Using stable-isotopelabeled internal standard or better chromatography could control matrix effect.

\section{Changing world of biomarkers in drug development}

Patrick Bennett of PPD (Richmond, VA, USA) gave a presentation on 'An overview of the changing world of biomarkers in drug development.' The report noted several challenges in biomarker analysis, including bioanalysis/characterization of biomarkers, from analytical, logistical and compliance standpoints and so on. The fit-for-purpose approach is commonly taken for biomarker analysis.

Robert Dodge of Bristol-Myers Squibb (Princeton, NJ, USA) presented the 'Crystal City VI biomarker meeting.' The lecture gave the background discussion of US FDA draft guidance requirement, which led to 
Crystal City VI workshop for biomarker development. Most biomarker measurements are relative quantitation with precision and accuracy assessment. CLSI guidelines warrant further review with attention to drug development objectives.

Jialin Mao of Genentech (South San Francisco, CA, USA) presented 'Biomarker challenges and innovative approaches to predict clinical outcomes.' The ideal CYP3A endogenous biomarker should reflect both intestinal and hepatic CYP3A allowing reflect CYP3A activity at baseline and reflect change in CYP3A activity following induction or inhibition. $4 \beta-\mathrm{HC}$ was valued as CYP3A biomarker due to its unique features. The report showed the potential applications of the hepatic CYP3A biomarker, like easy coordination with plasmas assay, possible utility in long-term treatment studies and in efficacy studies for chronic condition.

\section{Preparing for a successful first-in-human}

Marjoleen Nijsen of AbbVie (North Chicago, IL, USA) presented on 'Innovative translational modeling to build confidence in proof of concept (PoC).' The report noted the key success factors for building confidence in clinical proof of concept. The translation mindset should start early in drug discovery and should be organized alignment across functions. State-of-the-art toolbox, operational excellence and back-translation of clinical outcome ensured quality data generation and translational modeling, agile and effective decision making and future success separately.

Bianca Liederer of Genentech (South San Francisco, CA, USA) presented on 'Translation of preclinical in vivo and in vitro data to predict clinical exposures and drug-drug interactions for a novel NAMPT inhibitor'. NAMPT as a tumor metabolism target is essential for replenishment of NAD. GNE-617 is a novel and competitive inhibitor of NAMPT. Promising in vivo and in vitro results suggested GNE-617 is a potential development candidate.

Sylvia Zhao of Novartis (Shanghai, China) presented on 'Mechanism-based PK/PD modeling in drug development.' Several case studies were discussed, and mechanism-based PK/PD modeling was demonstrated to be a powerful tool to provide valuable information for decision making in the drug development process.

\section{Biomolecule bioanalysis}

Dongbei Li of WuXi AppTec (Shanghai, China) presented on 'Important considerations and case studies for anti-drug antibody assay development.' The report noted the importance of ADA detection approach, $\mathrm{ADA}$ and $\mathrm{Nab}$ assay format and assay validation. Con- siderations in drug tolerance, target interference, positive control selection, reagent storage and performance tracking and special issue in patient population were discussed.

Tianyi Zhang of Frontage (Shanghai, China) presented on 'Large molecule quantification by LC-MS and case studies.' Bioanalytical strategies to quantify large molecule drugs were discussed. In comparison with ligand-binding assays (LBA), LC-MS is shown to provide unique advantages for large-molecule drug quantification. The report noted the development of generic LC/MS PK assays for mAbs and ADCs, and consistency between ligand-binding assay results and LC/MS results was demonstrated. The application of LC HRMS for intact mAb quantification, as well as determination of free, total and conjugated ADCs, was illustrated in several case studies.

James Munday of Covance (Shanghai, China) presented on 'Analytical considerations for bi-specific antibodies and antibody drug conjugates.' It noted that no single package can be applied to all ADCs/bispecifics in diverse molecules, specificity, linkers and payloads. It also showed that the impact of immunogenicity on the mode of action can derisk the development pathway. A broad array of assays should be set in the analytical toolbox.

\section{Posters \& conference awards}

A total of 29 posters were presented, with comprehensive perspectives on industry-related issues and needs, firsthand experiences with specific applications and technologies shared by young scientists. The CPSA Shanghai meeting attracted many young scientists and researchers. To recognize their work and poster presentations, the meeting has set up Young Scientist Excellence Award and Innovator Award since year 2012. For this year, there were 11 Young Scientist Award recipients.

\section{Conclusion}

The 7th CPSA Shanghai conference has presented diverse topics of interest to drug researchers, focusing on the innovative approaches to reduce the attrition and predict clinical outcomes. As in the past, the conference was featured with highly interactive and diversified events, and has attracted attendees from overseas and local communities. This conference report summarized the key events at the conference to promote learning and sharing. Plans are already underway for next year's conference, CPSA Shanghai 2017. Gary Valaskovic of New Objective (Woburn, MA, USA) will serve as Program Chair and the meeting will be held on 12-14 April 2017 at the Renaissance Shanghai Pudong Hotel. For more details and information visit the conference website [2]. 


\section{Disclaimer}

$M$ Lee was the organizer of the CPSA series conference and K Dong was the program chair of the CPSA Shanghai 2016.

\section{Financial \& competing interests disclosure}

The authors have no relevant affiliations or financial involvement with any organization or entity with a financial

\section{References}

1 CPSA Shanghai 2016 website. www.cpsa-shanghai.com/2016/index.shtml interest in or financial conflict with the subject matter or materials discussed in the manuscript. This includes employment, consultancies, honoraria, stock ownership or options, expert testimony, grants or patents received or pending, or royalties.

No writing assistance was utilized in the production of this manuscript.

2 CPSA Shanghai website. www.cpsa-shanghai.com 